\title{
Intestinal Perforation Due to Foreign Body Ingestion in a Schizophrenic Patient
}

\author{
Mina Zarei, ${ }^{1}$ Behnam Shariati, ${ }^{2}$ and Reza Bidaki ${ }^{3,4}{ }^{*}$ \\ ${ }^{1}$ Department of Dermatology and Cutaneous Surgery, Miller School of Medicine, University of Miami, Miami, Florida, USA \\ ${ }^{2}$ Mental Health Research Center of Tehran, Iran University of Medical Sciences, Tehran, IR Iran \\ ${ }^{3}$ Research Center of Addiction and Behavioral Sciences, Shahid Sadoughi University of Medical Sciences, Yazd, IR Iran \\ ${ }^{4}$ Diabetes Research Center, Shahid Sadoughi University of Medical Sciences, Yazd, IR Iran \\ "Corresponding author: Reza Bidaki, Research Center of Addiction and Behavioral Sciences, Shahid Sadoughi University of Medical Sciences, Yazd, IR Iran. Tel/Fax: \\ +98-3532633555, E-mail: Reza_Bidaki@yahoo.com
}

Received 2015 May 21; Revised 2015 June 02; Accepted 2015 June 27.

\begin{abstract}
Introduction: Ingestion of foreign bodies has been previously reported in some patients with schizophrenia. This behavior may be a manifestation of delusional beliefs or a response to command hallucinations and can lead to severe complications.

Case Presentation: This paper reports a patient with schizophrenia who, as a manifestation of his illness, ingested a metallic skewer to kill ademon inside his abdomen that he believed was controlling him. As a result, he developed an acute intestinal perforation and underwent surgery.

Conclusions: It is of a great importance to closely monitor the therapy compliance of patients suffering from mental illnesses. This will benefit them by preventing some of the serious complications of their disease, which may include life-threatening conditions such as intestinal perforation that needs surgical intervention.
\end{abstract}

Keywords: Schizophrenia, Acute, Intestinal Perforation

\section{Introduction}

There are few reports regarding ingestion of foreign bodies in patients with schizophrenia (1-4). This behavior may be due to delusional beliefs or command hallucinations in these individuals and can cause severe complications $(5,6)$. Jacob et al. (7) reported the death of a schizophrenic woman due to aspiration after swallowing a large number of foreign bodies. Most swallowed foreign bodies pass through the gastrointestinal (GI) tract without causing any complications; however, GI perforation may occur in less than $1 \%$ of these cases, with the esophagus and ileo-cecum as the most susceptible areas (8). Here, we report a schizophrenic patient who, as a manifestation of his illness, ingested a metallic skewer based on his belief that it would kill ademon inside his body that was controlling him. He developed an acute intestinal perforation as a result.

\section{Case Presentation}

A 36-year-old single unemployed man who was first diagnosed with schizophrenia by a psychiatrist about 12 years ago is described here.
He remained in school until the eighth grade, when his abnormal behaviors at school caused him to stop his education. Also, for the same reason, he was fired from his job 2 years ago. He has been smoking cigarettes since 12years ago, and he started to smoke opium 11 years ago.

He did not have any evidence of brain damage or any major medical illnesses. He was the second child in a family of 6 with low socio-economic status. His parents were consanguineous and had no physical or mental abnormalities, although one of his younger brothers suffered from mental retardation. During the 12 years of his disease, he has been treated in both outpatient and inpatient settings. Due to exacerbation of his symptoms, he was admitted to a psychiatric ward on 4 occasions, and the course of his illness shows a fluctuating clinical picture. Since his first disease symptoms, he was diagnosed with personality disorder (borderline type), bipolar disorder, and even factitious disorder. His compliance with taking the prescribed medications and attending follow-ups has been very poor.

During the initial phase of his illness, he had prominent positive features, occasional violent outbursts, and delusions of persecution regarding his family and neighbors. All of his hospital admissions were due to psychotic exacerbations, which initially manifested as violent behav- 
ior and aggression towards others and then progressed to delusions of persecution, delusions of reference, and both auditory and visual hallucinations. During this phase of his disease, he was diagnosed with schizophrenia. He had a good appetite, but was suffering from early and terminal insomnia. He also had a history of self-mutilation from about ten years ago, but at the time of visit had no suicidal or homicidal thoughts or plans. His initial treatment plan included Valproic acid 200mg tablets TID (600 mg/day) and an Olanzapine tablet $5 \mathrm{mg}$ per day. Two years ago, this regimen was changed by his psychiatrist to Haloperidol $5 \mathrm{mg}$ BID (10 mg/day), Biperiden $2 \mathrm{mg} /$ day, Clonazepam 1 $\mathrm{mg} /$ day, Citalopram 20mg BID (40 mg/day), and Propranolol 10mg BID (20 mg/day) prescribed as oral medication. During his hospital admissions, he made steady recoveries, but his follow-ups and compliance were poor. All of his laboratory work-ups and brain computed tomography scans (CT scans) were normal and his electroencephalograms (EEG) showed no abnormalities.

During his fourth and last admission, he was brought to the emergency department because of severe abdominal pain. He had abdominal tenderness in his physical examination and his vital signs were stable. His abdominal $\mathrm{X}$-ray revealed the ingestion of a metallic skewer that was broken apart (Figure 1). After surgical consultation, endoscopic removal of the objects was suggested, but about an hour later the patient became medically unstable and had a board-like abdomen in his examination. After a second evaluation by a surgical team, the patient was diagnosed with intestinal perforation and an emergency exploratory laparotomy with enterotomy was performed. The surgery was without complications, and metallic items were removed from the patient's abdomen. He had an uneventful postoperative recovery.

After his recovery, at the subsequent psychiatric followup visit, he explained his motive for ingesting the metallic skewer and revealed that a demon that had been living inside his body had been controlling him for the previous three months. He insisted that the demon wouldn't let him tell the psychiatrists about its existence and wanted to control and use his soul to destroy the world. He believed that he could fight and kill the demon by ingesting the metallic skewer.

Two weeks after abdominal surgery, the patient attended our psychiatry outpatient clinic twice. He continued to have delusions of persecution and reference; however, he was convinced that because the surgery involved opening his abdomen, the demon had left his body and there was no need to fight with him anymore. Due to close monitoring by his family members, he did not ingest any further non-food items. Also, this close monitoring helped him have better compliance with his psychiatric visits and therapies.

\section{Discussion}

Although rare, it is very important to be aware of possible life-threatening medical conditions in patients suffering from mental illnesses. Basu et al. (2) reported a schizophrenic patient who ingested dead animals and 36 metallic items to become powerful by their effect, and this behavior resulted in bowel obstruction and laparotomy. In another report, Abraham and Alao described a schizophrenic inmate who ingested a can lid, which resulted in intestinal perforation (1).

Foreign object ingestion in psychotic patients is associated with highly repetitive behavior and high numbers of swallowed objects $(2,9)$. In other reported cases, ingestion of foreign bodies is often accompanied by command hallucinations (1). The uniqueness of our case is that the patient in our study wanted to destroy the commander, whom he believed was a demon in his body. He decided to ingest a metallic skewer to pursue this purpose. The patient was also suffering from delusions of persecution and both auditory and visual hallucinations.

This patient had normal brain function, brain CT scan, and EEG, and had no prior history of brain damage. Therefore, it can be presumed that his behavior was a direct manifestation of his schizophrenia.

The first-line recommended therapy after foreign body ingestion is to perform endoscopic removal of the object; however, surgical treatment is necessary when the object is sharp or is not removable by endoscopy, or when the patient develops an acute abdomen with bowel perforation, 10 as happened to our patient, which resulted in an emergency abdominal laparotomy.

There are not enough studies available that emphasize the biological underpinnings of foreign body insertion and ingestion behaviors. Therefore, physicians in all settings, especially primary care physicians, must be aware of this problem and make efforts to enhance their approach by early identification and proper management of such cases. In the presented case, the cause of the subject's abnormal eating behavior was psychiatric delusion, which could be prevented by closer monitoring of the patient and his compliance with the antipsychotic therapy. The authors' goal is to emphasize the importance of close monitoring of schizophrenic patients during their treatment to reach better compliance with the prescribed medications and follow-ups. Also, the role of the patients' family should not be underestimated, and they should be involved in the care of these individuals. 


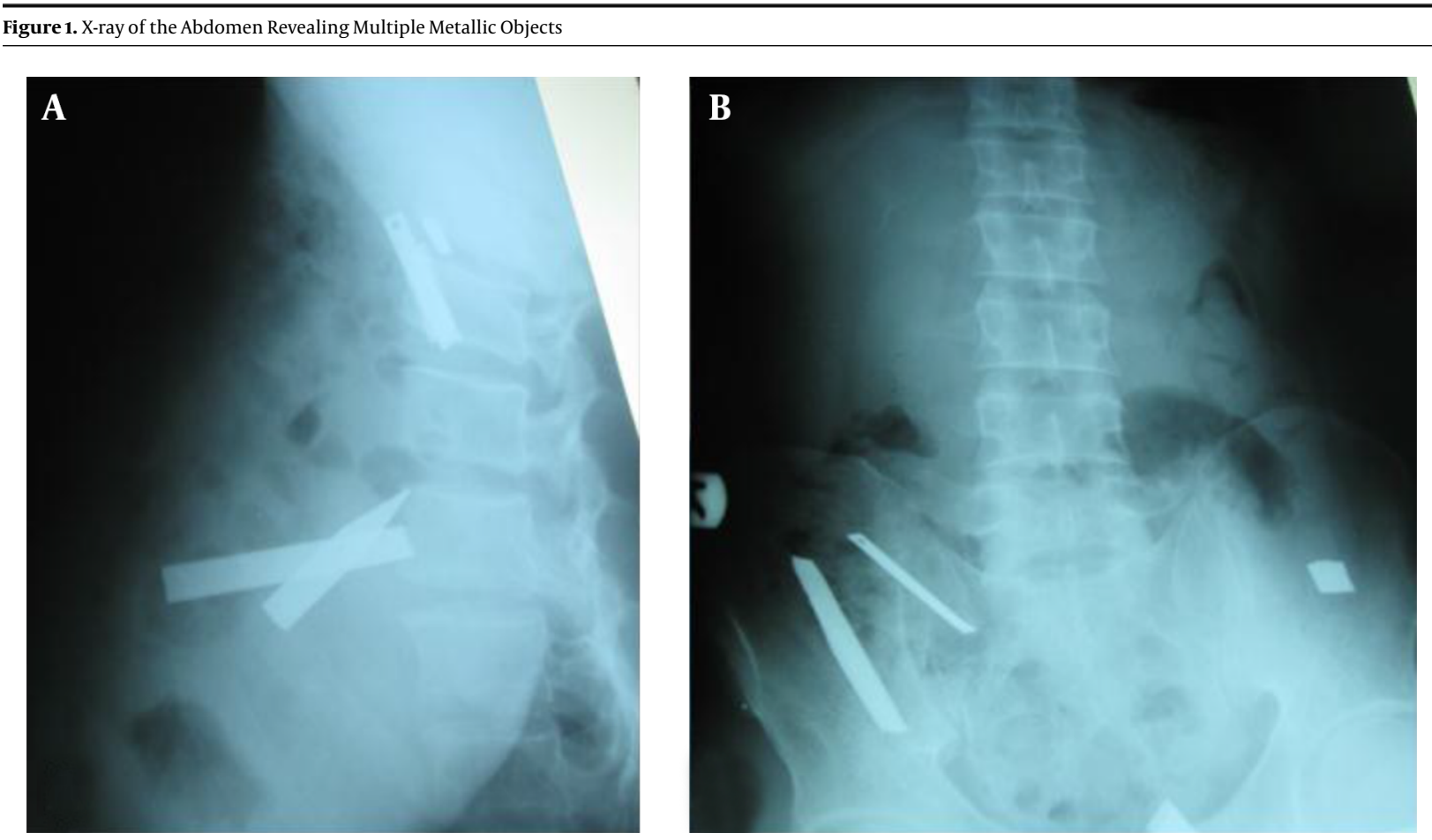

A, lateral view; B, AP view.

\section{Footnote}

Authors' Contribution: All the authors contributed to this manuscript. Mina Zarei wrote the manuscript. Behnam Shariati collected data and Reza Bidaki collected data and revised the manuscript and interviewed the patient.

\section{References}

1. Abraham B, Alao AO. An unusual foreign body ingestion in a schizophrenic patient: case report. Int J Psychiatry Med. 2005;35(3):3138. [PubMed: 16480246].

2. Basu S. Massive Ingestion of Foreign Bodies by Patients with Schizophrenia. Hong Kong J Psychiatr. 2003;13(2):26-8.

3. Han SY, McElvein RB, Aldrete JS. Compulsive ingestion of foreign bodies in a schizophrenic patient. South Med J. 1984;77(6):784-6. [PubMed: 6729562].
4. Subbotin VM, Davidov MI. [Rare observation of schizophrenic patient with inclination to swallow foreign bodies]. Zh Nevrol Psikhiatr Im S S Korsakova. 1998;98(4):66-7. [PubMed: 9606904].

5. Di Nunno N, Di Nunno C, Costantinides F, Garzya V, Bernasconi $\mathrm{P}$, Siciliano C, et al. Aorto-oesophageal fistula and intestinal infarction secondary to volvulus following ingestion of foreign bodies in a schizophrenic patient. Med Sci Law. 2000;40(4):350-7. [PubMed: 11281358].

6. Klein CA. Intentional ingestion and insertion of foreign objects: a forensic perspective. J Am Acad Psychiatry Law. 2012;40(1):119-26. [PubMed: 22396349].

7. Jacob B, Huckenbeck W, Barz J, Bonte W. Death, after swallowing and aspiration of a high number of foreign bodies, in a schizophrenic woman. Am J Forensic Med Pathol. 1990;11(4):331-5. [PubMed: 2275473].

8. Mehran A, Podkameni D, Rosenthal R, Szomstein S. Gastric perforation secondary to ingestion of a sharp foreign body. JSLS. 2005;9(1):91-3. [PubMed: 15791979].

9. Gitlin DF, Caplan JP, Rogers MP, Avni-Barron O, Braun I, Barsky AJ. Foreign-body ingestion in patients with personality disorders. Psychosomatics. 2007;48(2):162-6. doi: 10.1176/appi.psy.48.2.162. [PubMed: 17329611]. 